\title{
CONVEX SETS AND NEAREST POINTS. II ${ }^{1}$
}

\author{
R. R. PHELPS ${ }^{2}$
}

1. Introduction. In 1935 Motzkin [5] showed that if $S$ is a subset of the euclidean plane $E$ and $z \in S$ then the set $S_{z}$ of all points in $E$ having $z$ as a nearest point in $S$ is closed and convex. In [7] this result was easily extended to inner product spaces of arbitrary dimension. In $\$ 2$ of the present paper we show that any closed convex subset $T$ of a complete inner product space $E$ can be realized in such a manner, i.e. there exists a set $S$ and a point $z \in S$ such that $T=S_{z}$. Further, it is true that if $E$ is a normed linear space of dimension at least three then every closed convex subset $T$ can be so realized only if $E$ is a complete inner product space.

If $A$ is a subset of a normed linear space $E$ and $x, y$ are in $E$ we say that $y$ is point-wise closer to $A$ than is $x$ provided $\|y-a\|<\|x-a\|$ for each $a \in A$. If $x$ is such that no point of $E$ is point-wise closer to $A$ than is $x$ we call $x$ a closest-point to A. Fejér [1] has noted that in the euclidean plane the set $C(A)$ of all closest points to $A$ is precisely $K(A)$, the closed convex hull of $A$. In applying this result, Fejér, and later Motzkin and Schoenberg [6], actually used a weaker version, which we will call property (F): If $A \subset E$, then $C(A) \subset K(A)$. In $\S 3$ we show that property $(\mathrm{F})$ characterizes complete inner product spaces of dimension at least three, while in two-dimensional spaces it is equivalent to strict convexity. We also extend Fejér's characterization of $K(A)$ to complete inner product spaces and show that it holds in strictly convex two-dimensional spaces if $A$ is bounded.

2. Nearest-point sets. If $S$ is a subset of a normed linear space $E$ and if $z \in S, S_{z}$ will denote the set $\left\{x:\|x-z\|=\inf _{y \in S}\|x-y\|\right\}$ of all points in $E$ whose distance from $S$ is attained at $z$. Using the alternative formulation $S_{z}=\{x:\|x-z\| \leqq\|x-y\|$ for each $y \in S\}$ it is easy to see that $S_{z}$ is always closed. In several papers $[2 ; 3 ; 4]$ James has successfully exploited a concept of orthogonality which is defined as follows: We say that $x$ is orthogonal to $y$ (and write $x \perp y$ ) if $\|x\|$ $\leqq\|x-\lambda y\|$ for each $\lambda \in R$, where $R$ is the real numbers. Note that this is equivalent to saying that $x$ has the origin $\phi$ as a nearest point

Presented to the Society February 23, 1957; received by the editors July 12, 1957, and, in revised form, May 16, 1958.

${ }^{1}$ Work on this paper sponsored in part by the Office of Ordnance Research, U. S. Army, under contract DA-04-200-ORD-292.

2 The author wishes to express his appreciation to Professor V. L. Klee for having read and criticized this paper. 
in $R y=\{\lambda y: \lambda \in R\}$, the line determined by $y$ and $\phi$ (assuming $y \neq \phi)$-in other words, $x \in(R y)_{\phi}$. That this is a generalization of the usual inner product space concept of orthogonality is easily verified, using the identity

$$
\|x-\lambda y\|^{2}=\|x\|^{2}-2 \lambda(x, y)+\|y\|^{2}
$$

by merely showing that in such a space $x \perp y$ if and only if $(x, y)=0$. If $L$ is a linear subset of $E$ we write $L \perp y(y \perp L)$ provided $x \perp y(y \perp x)$ for each $x \in L$, and if $M$ is linear, $L \perp M$ is defined in a similar way. Note that $x \perp y$ if and only if $R x \perp R y$.

James has proved a theorem [4, Theorem 4] which, after a slight extension, will be quite useful to us.

Theorem 1 (James). Suppose $E$ is a normed linear space of dimension at least three. Then $E$ is a complete inner product space provided each hyperplane through the origin is orthogonal to some point $x \neq \phi$.

Proof. James has shown that the condition is sufficient to imply that $E$ is an inner product space, so we need only show that $E$ is also complete. Suppose $y \in F$, where $F$ is the completion of $E$. Then the set $H=\{z: z \in F$ and $(z, y)=0\}$ is a hyperplane in $F$ and $H_{0}=H \cap E$ is a hyperplane in $E$ which contains $\phi$. By hypothesis there exists $x \neq \phi$ such that $H_{0} \perp x$. But, as noted previously, this implies that $(z, x)=0$ for each $z \in H_{0}$. Hence, by continuity of the inner product and the fact that $H_{0}$ is dense in $H$, we have $(z, x)=0$ for each $z \in H$. This implies that $y \in R x \subset E$, so that $E=F$ is complete.

Note that if $H$ is a hyperplane in an inner product space and $x$ is a point of least norm in $H$ then $x \in(H-x)_{\phi}$ or $x \perp H-x$.

We call a subset $T$ of a normed linear space $E$ a nearest-point set if there exists a set $S \subset E$ and a point $z \in S$ such that $T=S_{z}$. In [7] it was shown that every nearest-point set in $E$ is closed and convex if and only if $E$ is an inner product space. Here we consider the question: For what class of normed linear spaces is every closed convex set a nearest-point set? This is partially answered by the following theorem.

TheOREM 2. In a complete inner product space $E$ every closed convex set $T$ is a nearest-point set.

Proof. If $T=E$, let $S=\{\phi\}$. Otherwise, $T=\bigcap_{H \in \mathcal{F C}} H^{\prime}$ where $\mathfrak{H C}$ is the collection of all hyperplanes $H$ such that $H$ determines a closed half-space $H^{\prime}$ for which $T \subset H^{\prime}$. Pick any point of $T$-we will suppose that it is the origin. For $H \in \mathcal{H C}$ let $x_{H}$ be the point of least norm in $H$ and define $S=\left\{2 x_{H}: H \in \mathfrak{F}\right\} \cup\{\phi\}$. Since $x_{H} \perp H-x_{H}$ for each 
$H \in \mathfrak{F}$, we have $H^{\prime}-x_{H}=\left\{y:\left(x_{H}, y\right) \leqq 0\right\}$ or $H^{\prime}=\left\{y:\left(x_{H}, y-x_{H}\right)\right.$ $\leqq 0\}$. But, letting $z=y-x_{H},\left(z, x_{H}\right) \leqq 0$ if and only if $2\left(z, x_{H}\right)$ $\leqq-2\left(z, x_{H}\right)$ which is in turn equivalent to $\|z\|^{2}+2\left(z, x_{H}\right)+\left\|x_{H}\right\|^{2}$ $\leqq\|z\|^{2}-2\left(z, x_{H}\right)+\left\|x_{H}\right\|^{2}$. This, however, is the same as $\left\|z+x_{H}\right\|^{2}$ $\leqq\left\|z-x_{H}\right\|^{2}$ or $\|y\|^{2} \leqq\left\|y-2 x_{H}\right\|^{2}$. Thus, $H^{\prime}=\left\{y:\|y\| \leqq\left\|y-2 x_{H}\right\|\right\}$ and hence $T=\bigcap_{H \in \mathcal{F}} H^{\prime}=\left\{y:\|y\| \leqq\left\|y-2 x_{H}\right\|\right.$ for each $\left.H \in \mathcal{F C}\right\}=\{y:\|y\|$ $\leqq\|y-z\|$ for each $z \in S\}=S_{\phi}$.

The above proof shows that for any $z \in T$ there exists a set $S(T, z)$ such that $T=S(T, z)_{z}$. Applying the (constructive) method of the proof to a specific example we find, with the aid of some elementary analytic geometry, that if $T$ is the closed disc bounded by the curve $r=2 \cos \theta$ then $S(T, \phi)$ is the area not inside the cardioid $r$ $=2(1+\cos \theta)$. Note that we could omit from $S$ any point not on the cardioid and still have $T=S_{\phi}$. We can, however, show that in a certain sense the set $S$ can be taken to be unique. Suppose that $T$ is a nearest-point subset of a normed linear space $E$; then there exists $S \subset E$ and $z \in S$ such that $T=S_{z}$. Let $\delta$ be the collection of all sets $S$ containing $z$ such that $S_{z}=T$. Then, letting $Q(T, z)=\bigcup_{S \in \mathcal{S}} S$, we see that $Q(T, z)_{z}=\bigcap_{S \in \mathcal{S}} S_{z}=T$ so that $Q(T, z) \in \mathcal{S}$ and is the biggest member of $\delta$. Henceforth, if a set $T$ is a nearest-point set, $Q(T, z)$ will denote the set defined above. If there is no chance for confusion we will simply denote it by $Q$.

Suppose that $T=S_{z}$ for some $S$ and $z \in S$. Then $Q(T, z)$ $=\{x:\|x-y\| \geqq\|z-y\|$ for each $y \in T\}$. For clearly $x \in Q$ implies $\|x-y\| \geqq\|z-y\|$ for each $y \in T$; and if this latter is true, $T \subset[Q \cup\{x\}]_{z} \subset Q_{z}=T$ so that $[Q \cup\{x\}]_{z}=T$ and by the maximality property of $Q, x \in Q \cup\{x\} \subset Q$. Using this description of $Q$ it is easy to see that $Q$ is closed. Further, the set $S$ constructed in the proof of Theorem 2 is actually equal to $Q(T, \phi)$, for $T=S_{\phi}$ implies that $S \subset\{x:\|x-y\| \geqq\|y\|$ for each $y \in T\}$. Suppose that $x$ is a point of the latter set, then the hyperplane $H=\{w:\|w-x\|=\|w\|\}$ passes through $(1 / 2) x$ and is orthogonal to $R x$. Hence, if $H^{\prime}$ is the closed half-space determined by $H$ which contains $\phi, y \in T$ implies $\|y\| \leqq\|y-x\|$ so that $y \in H^{\prime}$ and thus $T \subset H^{\prime}$. We have, then, that $H \in \mathcal{F}$ and that $x$ is of the form $2 x_{H}$ and is therefore in $S$.

It is natural to wonder if $Q$ may be convex. Suppose that $T=Q_{\phi}$ and that $Q$ is convex, then by Lemmas 3.1 and 4.1 of [7] $T$ must be a convex cone with vertex $\phi$. Using identity (1) it is easily verified that whenever $T$ is a cone with vertex $\phi, Q=\{x:(x, y) \leqq 0$ for each $y \in T\}$ and hence $Q$ is also a convex cone with vertex $\phi$ (the dual or polar cone of $T$ [8]). Thus, $Q$ is convex if and only if both $T$ and $Q$ are convex cones with common vertex. 
In what follows we show that the hypothesis in Theorem 2 that $E$ be a complete inner product space is necessary, if the dimension of $E$ is at least three.

Theorem 3. Suppose that $E$ is a normed linear space of three or more dimensions and that every closed convex subset of $E$ is a nearest-point set. Then $E$ is a complete inner product space. ${ }^{3}$

Proof. Suppose $H$ is a hyperplane in $E$ which passes through the origin. By Theorem 1 we can conclude that $E$ is a complete inner product space provided every such hyperplane is orthogonal to some $x \neq \phi$, i.e. provided $H \perp R x$ for some $x \neq \phi$. Now, $H$ is closed and convex so there exists a set $S$ and a point $z \in S$ such that $I=S_{z}$. By the preceding discussion there exists a maximal set $Q(H, z)$ such that $H=Q_{z}$. If $Q=\{z\}, Q_{z}=H$ would be the entire space, contradicting the fact that $H$ is a hyperplane. Pick $x \in Q \sim\{z\}$ and suppose $y \in H$ and $\lambda \in R \sim\{0\}$. Since $z \in H$, there exists $w \in H$ such that $y=\lambda(w-z)$. We know that $\|w-x\| \geqq\|w-z\|$ hence $\|\lambda(w-x)\| \geqq\|\lambda(w-z)\|$ or $\|y-\lambda(x-z)\| \geqq\|y\|$. Therefore $H \perp R(x-z)$, which was to be shown.

What if $E$ is two-dimensional and every closed convex subset of $E$ is a nearest-point set? By somewhat lengthy arguments we have been able to show that this implies strict convexity of $E$, and that $E$ is an inner product space provided we assume that $E$ is smooth. We have been unable to show that the assumption of smoothness is necessary. This result indicates, however, that strict convexity alone does not imply that every closed convex subset of $E$ is a nearest-point set, for such an implication would in turn imply the false statement that every smooth and strictly convex two-dimensional normed linear space is an inner product space.

3. Convex sets and closest points. We first prove Fejér's theorem (see introduction) in a more general setting.

THEOREM 4 (FEJÉR). If $A$ is a subset of a complete inner product space $E$, the closed convex hull $K(A)$ of $A$ is the set $C(A)$ of closest points to $A$.

Proof. Suppose $x \notin K(A)$, then there exists a hyperplane $H$ separating $x$ from $K(A)$ such that $x \in H$. Since $E$ is complete we know that the distance from $x$ to $H$ is attained at some (unique) point of $H$. Without loss of generality we may assume that this point is the origin, so that $\|x\| \leqq\|x-z\|$ for each $z \in H$. This says, then, that $x \perp H$ and hence $H \perp x$. Thus, if $a \in A$, there exists $z \in H \cap[a, x]$. Now,

${ }^{3}$ We wish to thank the referee for suggesting changes which simplified the proof of this theorem, as well as the proof of Theorem 5 . 
$\|z\|<\|z-x\|$ and therefore $\|a\| \leqq\|a-z\|+\|z\|<\|a-z\|+\|z-x\|$ $=\|a-x\|$. Hence $\phi$ is point-wise closer to $A$ than is $x$, giving $x \in C(A)$. Suppose $x \notin C(A)$, then there exists $y \in E$ which is point-wise closer to $A$ than is $x$. The set $H=\{z:\|z-y\|=\|z-x\|\}$ is a hyperplane through $(1 / 2)(x+y)$ which determines a closed half-space $H^{\prime}$ $=\{z:\|z-y\| \leqq\|z-x\|\}$ containing $A$. Hence $K(A) \subset H^{\prime}$ and since $x \notin H^{\prime}, x \notin K(A)$.

Defining property $(\mathrm{F})$ as in the introduction, we now determine the class of spaces possessing the property.

Theorem 5. Suppose that $E$ is a normed linear space of dimension at least three which possess property $(\mathrm{F})$. Then $E$ is a complete inner product space.

Proof. We again make use of Theorem 1. Suppose that $H$ is a hyperplane through $\phi$. If $x \notin H=K(H)$ then by assumption $x \notin C(H)$ and there must exist a point $y$ such that $\|h-y\|<\|h-x\|$ for each $h \in H$. The line $L$ determined by $x$ and $y$ must hit $H$ at $w$, say. (For $\phi$ has a nearest point $z$ in $L$, so if $L$ were parallel to $H, h=x-z$ would be in $H$ and have $x$ as a nearest-point in $L$. In particular, $h$ would not be closer to $y \in L$ than to $x$, a contradiction.) Now, $w=\alpha y+(1-\alpha) x$ for some $\alpha \neq 0$, so if $\lambda \neq 0$ and $h$ is any point of $H$ we have $\alpha \lambda^{-1} h$ $+w \in H \quad$ and $\|h-\lambda(x-y)\|=\left\|\lambda \alpha^{-1}\left[\left(\alpha \lambda^{-1} h+w\right)-x\right]\right\|$ $>\left\|\lambda \alpha^{-1}\left[\left(\alpha \lambda^{-1} h+w\right)-y\right]\right\|=\|h+\beta \lambda(x-y)\|$, where we have let $\beta$ $=\alpha^{-1}(1-\alpha)$. If $\beta=0$ we have the inequality we are seeking; otherwise, we use the fact that $h \in H$ if and only if $\beta^{-1} h \in H$ to conclude, by induction, that $\|h-\lambda(x-y)\|>\left\|h+\beta^{n} \lambda(x-y)\right\|$ for each positive integer $n$. Setting $h=\phi$ shows that $|\beta|<1$, so, taking the limit as $n \rightarrow \infty$, we have $\|h-\lambda(x-y)\|>\|h\|$. This shows that $H \perp(x-y)$ and hence we can conclude from Theorem 1 that $E$ is a complete inner product space.

Lemma 6. If a normed linear space $E$ has property $(\mathrm{F})$ then $E$ is strictly convex.

Proof. If $E$ is not strictly convex there exist points $x, y$ in $E$ such that the distance from $y$ to each point of the segment $[-x, x]$ is exactly 1. Let $A=[-y, y]$; then $x \notin A=K(A)$ and hence $x \notin C(A)$, by hypothesis. There must exist a point $z$ in $E$ which is point-wise closer to $A$ than is $x$. Thus, $\|z-y\|<\|x-y\|=1$ and $\|z+y\|<\|x+y\|$ $=1$ so $2=\|2 y\|=\|(-y)-y\| \leqq\|-y-z\|+\|z-y\|<2$, a contradiction.

THeORem 7. Suppose that $E$ is a two-dimensional normed linear space. Then $E$ possesses property $(\mathrm{F})$ if and only if it is strictly convex.

Proof. Lemma 6 proves the necessity. Suppose $E$ is strictly con- 
vex and $A \subset E$. If $x \notin K(A)$ there exists a line $L$ separating $K(A)$ from $x$ such that $x \notin L$. We can suppose that $\phi \in L$ and that $L=R y$ for some $y \neq \phi$. There exists a line $M$ through $\phi$ such that $y \perp M$ [3, Theorem 2.2], so $M+x$ hits $R y$ at $\alpha y$ for some $\alpha \in R$. We will show that $\alpha y$ is point-wise closer to $A$ than is $x$. Suppose $a \in A$, then $[a, x]$ hits $R y$ at $\beta y$, say. Now $M+x=M+\alpha y$ so $x-\alpha y \in M \sim\{\phi\}$ and hence, by strict convexity, $\|\beta y-\alpha y\|<\|(\beta y-\alpha y)-(x-\alpha y)\|$ $=\|\beta y-x\|$. Then

$\|a-\alpha y\| \leqq\|a-\beta y\|+\|\beta y-\alpha y\|<\|a-\beta y\|+\|\beta y-x\|=\|a-x\|$.

Hence $x \notin C(A)$ and we have $C(A) \subset K(A)$.

In stating his theorem Fejér assumed that $A$ was compact. This is an unnecessary restriction if we wish to prove his characterization of $K(A)$ in the euclidean plane, but it is interesting to note that if we merely assume that $A$ is bounded we can prove his characterization in any two-dimensional strictly convex normed linear space.

Theorem 8. Suppose that $A$ is a bounded subset of a strictly convex two-dimensional normed linear space $E$. Then $K(A)=C(A)$.

Proof. That $C(A) \subset K(A)$ follows from the strict convexity and Theorem 7. Suppose that $x \notin C(A)$, then there exists a point $y \in E$ such that $\|y-a\|<\|x-a\|$ for each $a \in A$. We can suppose without loss of generality that $x=\phi$. Now there exists a point $z \neq \phi$ such that $z \perp y$ [3] and hence $L \perp y$ for $L=R z$. Letting $L^{\prime}$ be the closed halfspace determined by $L$ which does not contain $y$, we see that $\operatorname{cl} A$, the closure of $A$, is contained in $E \sim L^{\prime}$ as follows: Let $S=\{w:\|w-y\|$ $\leqq\|w\|\}$; then $S$ is closed, contains $A$ and hence contains $\operatorname{cl} A$. Furthermore, if $w$ is a point of $L^{\prime}$, we can use strict convexity and the triangle inequality (as in the proof of Theorem 7) to show that $\|w\|<\|w-y\|$. Thus, $w \notin S$ so $S \subset E \sim L^{\prime}$ and $\mathrm{cl} A \subset E \sim L^{\prime}$.

Since $A$ is bounded, $\operatorname{cl} A$ is compact and hence $\operatorname{conv} \operatorname{cl} A$, the convex hull of $\mathrm{cl} A$, is closed. Thus, $K(\operatorname{cl} A)=\operatorname{conv} \operatorname{cl} A$ and since $E \sim L^{\prime}$ is convex, conv cl $A \subset E \sim L^{\prime}$. We have, then, that $K(A)$ $\subset K(\mathrm{cl} A) \subset E \sim L^{\prime}$, so that $\phi$, being in $L \subset L^{\prime}$, is not in $K(A)$. Therefore $C(A)=K(A)$ for any bounded set $A$.

It is possible to construct an example in which the above result fails for an unbounded set $A$.

\section{BiBLIOGRAPHY}

1. L. Fejêr, Über die Lage der Nullstellen von Polynomen, die aus Minimumforderungen gewisser Art entspringen, Math. Ann. vol. 85 (1922) pp. 41-48.

2. R. C. James, Orthogonality in normed linear spaces, Duke Math. J. vol. 12 (1945) pp. 291-302. 
3. - Orthogonality and linear functionals, Trans. Amer. Math. Soc. vol. 61 (1947) pp. 265-292.

4. ㄴ. Inner products in normed linear spaces, Bull. Amer. Math. Soc. vol. 53 (1947) pp. 559-566.

5. Th. Motzkin, Sur quelques propriétés caractêristiques des ensembles bornés non convexes, Atti Accad. Naz. Lincei. Rend. 6 vol. 21 (1935) pp. 773-779.

6. Th. Motzkin and I. J. Schoenberg, The relaxation method for linear inequalities, Canad. J. Math. vol. 6 (1954) pp. 393-404.

7. R. R. Phelps, Convex sets and nearest points, Proc. Amer. Math. Soc. vol. 8 (1957) pp. 790-797.

8. L. Sandgren, On convex cones, Math. Scand. vol. 2 (1954) pp. 19-28.

UNIVERSITY OF WASHINGTON

\section{NOTE ON PRODUCTS IN Ext}

\section{NOBUO YONEDA ${ }^{1}$}

The objective of this note is to present an interrelation between the $V$-product in [1] and the composition product in $[2 ; 3]$, which in turn gives a comparison of the cup-product with the composition product. Similar relations can be obtained also for other products involving Tor and (iterated) connecting homomorphisms.

We retain the notations in $[1$, Chapter XI]. The external product $\mathrm{V}: \operatorname{Ext}_{\Lambda \otimes \Sigma^{*}}(A, C) \otimes \operatorname{Ext}_{\Sigma \otimes \Gamma^{*}}\left(A^{\prime}, C^{\prime}\right) \rightarrow \operatorname{Ext}_{\Lambda \otimes \Gamma^{*}}\left(A \otimes_{\Sigma} A^{\prime}, C \otimes_{\Sigma} C^{\prime}\right)$ is defined in the situation $\left({ }_{\Lambda} A_{\Sigma},{ }_{\Delta} C_{\Sigma},{ }_{\Sigma} A_{\Gamma}^{\prime},{ }_{\Sigma} C_{\Gamma}^{\prime}\right)$ under the following assumption: (i) $\Lambda, \Gamma, \Sigma$ are $K$-projective; (ii) $\operatorname{Tor}_{n}^{\Sigma}\left(A, A^{\prime}\right)=0$ for $n>0$. To this situation we now add $\left({ }_{\Lambda} B_{\Sigma},{ }_{\Sigma} B_{\Gamma}^{\prime}\right)$ and $\left(\mathrm{ii}^{\prime}\right) \operatorname{Tor}_{n}^{\Sigma}\left(B, B^{\prime}\right)$ $=0$ for $n>0$. For $a \in \operatorname{Ext}_{\Lambda \otimes \Sigma^{*}}^{p}(A, B)$ and $b \in \operatorname{Ext}_{\Lambda \otimes \Sigma^{*}}^{q}(B, C)$ the composition product $b \circ a$ lies in $\operatorname{Ext}_{\Lambda \otimes \Sigma^{*}}^{p+q}(A, C)$. For $a^{\prime} \in \operatorname{Ext}_{\Sigma}^{p^{\prime}} \otimes \Gamma^{*}\left(A^{\prime}, B^{\prime}\right)$ and $b^{\prime} \in \operatorname{Ext}_{\Sigma}^{q^{\prime}} \otimes \Gamma^{*}\left(B^{\prime}, C^{\prime}\right), b^{\prime} \circ a^{\prime}$ lies in $\operatorname{Ext}_{\Sigma}^{p^{\prime}+q^{\prime}} \otimes \Gamma^{*}\left(A^{\prime}, C^{\prime}\right) .(b \circ a=a \circ b$ in the notation of [2].)

Proposition 1. $(b \circ a) \bigvee\left(b^{\prime} \circ a^{\prime}\right)=(-1)^{p q^{\prime}}\left(b \bigvee b^{\prime}\right) \circ\left(a \bigvee a^{\prime}\right)$.

In fact let $X, Y$ be $\Lambda \otimes \Sigma^{*}$-projective resolutions of $A, B$ respectively, and let $X^{\prime}, Y^{\prime}$ be $\Sigma \otimes \Gamma^{*}$-projective resolutions of $A^{\prime}, B^{\prime}$. Then $X \otimes_{\Sigma} X^{\prime}, Y \otimes_{\Sigma} Y^{\prime}$ are $\Lambda \otimes \Gamma^{*}$-projective resolutions of $A \otimes_{\Sigma} A^{\prime}, B \otimes_{\Sigma} B^{\prime}$. We consider these resolutions as chain complexes with 0 's in negative dimensions. Suppose that $a, b, a^{\prime}, b^{\prime}$ are respectively represented by maps $\alpha: X_{p} \rightarrow B, \beta: Y_{q} \rightarrow C, \alpha^{\prime}: X_{p^{\prime}}^{\prime} \rightarrow B^{\prime}$, and $\beta^{\prime}: Y_{q^{\prime}}^{\prime} \rightarrow C^{\prime}$. The map $\alpha$

Received by the editors April 25, 1958.

${ }^{1}$ Work done while the author was engaged under contract NONR 2383. 